\title{
Degradação rápida de folhas de policarbonato
}

\section{Flash degradation of polycarbonate sheets}

Francisco Marcone Lima ${ }^{1}$, Felipe Mota Martins ${ }^{1}$, João Paulo Costa do Nascimento², Paulo Herbert França Maia Júnior ${ }^{1,3}$, Ana Fabíola Leite Almeida ${ }^{1}$, Francisco Nivaldo Aguiar Freire ${ }^{1}$, Enio Pontes de Deus ${ }^{3}$

\section{RESUMO}

A degradação térmica de folhas de policarbonato foi feita por pulverização pirolítica da solução aquosa de hidróxido de sódio. Para a pulverização da solução foi usado um bico com vácuo. A espectroscopia de fotoluminescência, a microscopia eletrônica de varredura (MEV) e a fluorescência de raios-X (FRX) foram as técnicas usadas para a caracterização do material, antes e após a degradação. As folhas de policarbonato com e sem degradação quando expostas à radiação na faixa de comprimento de onda ultravioleta da ordem de $385 \mathrm{~nm}$ exibiram fotoluminescência. Os picos de emissões ocorreram em $410 \mathrm{~nm}$ e $440 \mathrm{~nm}$, faixas do violeta e azul, respectivamente. Adicionalmente, com o auxílio das demais técnicas, é proposta uma possível razão para os resultados mostrados pela espectroscopia de fotoluminescência.

Palavras-chave: Policarbonato; Degradação; Vácuo; Fotoluminescência.

\begin{abstract}
The thermal degradation of polycarbonate sheets was made from water-sodium hydroxide solution by spray pyrolysis. It was created a vacuum within nozzle for provides the spray. The material, before and after of the thermal degradation, were characterized by photoluminescence spectroscopy, scanning electron microscopy and X-ray fluorescence. Polycarbonate sheets, before and after of the thermal degradation, when subjected to monochromatic ultraviolet radiation with a wavelength of $385 \mathrm{~nm}$ showed photoluminescence. It was observed that the emission occurred in the violet band and the blue band, with peak intensities at $410 \mathrm{~nm}$ and $440 \mathrm{~nm}$, respectively. In addition, the other techniques help in understanding on the results obtained from photoluminescence spectroscopy.
\end{abstract}

Keywords: Polycarbonate; Degradation; Vacuum; Photoluminescence.

'Universidade Federal do Ceará - Centro de Tecnologia - Laboratório de Filmes Finos e Energias Renováveis - Fortaleza (CE) - Brasil

2Universidade Federal do Ceará - Centro de Ciências - Laboratório de Telecomunicações, Ciência e Engenharia de Materiais - Fortaleza (CE) - Brasil ${ }^{3}$ Universidade Federal do Ceará - Centro de Tecnologia - Laboratório de Mecânica da Fratura e Fadiga - Fortaleza (CE) - Brasil

Autor correspondente: Francisco Marcone Lima - Universidade Federal do Ceará - Centro de Tecnologia - Laboratório de Filmes Finos e Energias Renováveis - Campus do Pici - Bloco 714 - CEP 60455-760 - Fortaleza (CE) - Brasil - E-mail: marconeufc@gmail.com

Recebido:16/08/2016 Aprovado: 11/01/2017 


\section{INTRODUÇÃO}

O polímero policarbonato (PC) é um material termoplástico que pode ser dividido em duas classes estruturais: alifáticos e $\operatorname{aromáticos}^{(1)}$. Dentre as várias aplicações deste material estão substituição do vidro em janelas, fabricação de CD e DVD e uso em unidades de biomonitoramento em análise de água ${ }^{(1,2)}$. A larga faixa de aplicação é devido ao fato do PC ser um importante material de engenharia com propriedades muito úteis, tais como capacidade calorífica elevada, alta transparência, boa estabilidade térmica, elevada resistência mecânica e estabilidade química à radiação ultravioleta ${ }^{(1-3)}$.

Em relação aos polímeros, inúmeras condições às quais eles são submetidos podem levar à sua degradação e à perda de suas propriedades $^{(4)}$. A degradação por efeito térmico, em particular, é de interesse crescente e seus efeitos têm sido estudados por microscopia eletrônica de varredura $(\mathrm{MEV})^{(2)}$. Em particular, quando o PC é imerso em solução aquosa de hidróxido de sódio e o sistema é submetido a faixa de temperatura de $40^{\circ} \mathrm{C}$ a $60^{\circ} \mathrm{C}$, há degradação do PC com geração dos subprodutos bisfenol A (BFA) e carbonato de sódio $\left(\mathrm{Na}_{2} \mathrm{CO}_{3}\right)^{(3)}$.

Apesar de a propriedade fotoluminescente ter sido relatada para materiais a base de carbono, como dots de carbono ${ }^{(5-10)}$, não há estudos sobre a capacidade do PC de converter radiação ultravioleta em luz visível através da fotoluminescência. Também, ainda não há divulgação de estudos sobre os efeitos de névoa cáustica sobre o PC. A partir deste contexto, este trabalho tem como objetivo reportar o efeito da degradação térmica via névoa cáustica sobre a propriedade do PC de converter radiação ultravioleta em luz visível. As informações obtidas sobre a estabilidade térmica podem ser úteis em estudos para novas aplicações PC.

\section{MATERIAIS E MÉTODOS}

As soluções aquosas com $50 \mathrm{~mL}$ de $\mathrm{NaOH} 0.1 \mathrm{~N}$ (marca Dinâmica) foram submetidas a vácuo ( 0,7035 bar) com auxílio de bico para gerar névoas cáusticas que foram aspergidas sobre as folhas de PC, nas temperaturas ambiente e de $50^{\circ} \mathrm{C}$. O sistema foi montado numa capela e o exaustor ficou ligado durante todo o trabalho. O sistema de pulverização pirolítica foi composto por um bico (APREX's alfa 4) conectado a um recipiente para armazenar a solução e por um compressor de ar (Twister SHULZ) ${ }^{(11)}$. Também, um soprador térmico (Bosch GHG 630 DCE) e termopar tipo K foram usados.

Antes e depois da degradação, as folhas de PC foram lavadas com água deionizada e submetidas a técnicas de caracterização. As caracterizações foram realizadas via espectroscopia de fotoluminescência (DPSSL DRIVER Modelo LD-WL206) do Laboratório de Telecomunicações e Ciência e Engenharia de Materiais (LOCEM), MEV e EDS (TM3000 - HITACH) do Laboratório de Microscopia (LAMEV) e fluorescência de raios-X (ZSXMini II RIGAKU) do Laboratório de Raios-X (LRX). Todos pertencentes à Universidade Federal do Ceará.

\section{RESULTADOS E DISCUSSÃO}

Há diversas condições nas quais os polímeros são submetidos que levam a sua degradação(4). O gás de arrasto (ar) usado neste trabalho gera vácuo necessário para formar a névoa cáustica de forma rápida e barata. Através deste sistema é possível simular algumas condições de intempéries, tais como de chuva ácida ou básica e também a umidade do ar, nas quais as folhas de PC estariam submetidas se expostas ao ambiente. A metodologia adotada neste trabalho pode ser adaptada e melhorada para tornar as condições mais próximas das condições de intempéries reais.

Folhas de PC têm sido usadas em ensaios de degradação térmica com exposição do material a pulverização com água e iluminação por 4 horas a temperaturas de $55^{\circ} \mathrm{C}$ e $12^{\circ} \mathrm{C}^{(2)}$. Neste trabalho foi realizada uma degradação rápida, cerca de 20 minutos, usando hidróxido de sódio, água e gás de arrasto para gerar névoa cáustica (meio básico) com duas condições de temperatura (ambiente e $50^{\circ} \mathrm{C}$ ). Para as condições adotadas, somente à $50^{\circ} \mathrm{C}$ houve degradação das folhas do polímero PC. Após a decomposição térmica, as amostras apresentaram incrustações. As amostras foram lavadas com água deionizada para remoção de impurezas solúveis antes de serem analisadas utilizando as técnicas MEV, EDS e FRX.

A variação das propriedades do PC tem sido usada como um indicativo de que o material sofreu algum tipo de degradação ${ }^{(1,2)}$. As imagens obtidas com auxílio de MEV ilustram a morfologia superficial da folha de PC antes (Fig. 1a) e depois (Fig. 1b) da

\section{A}

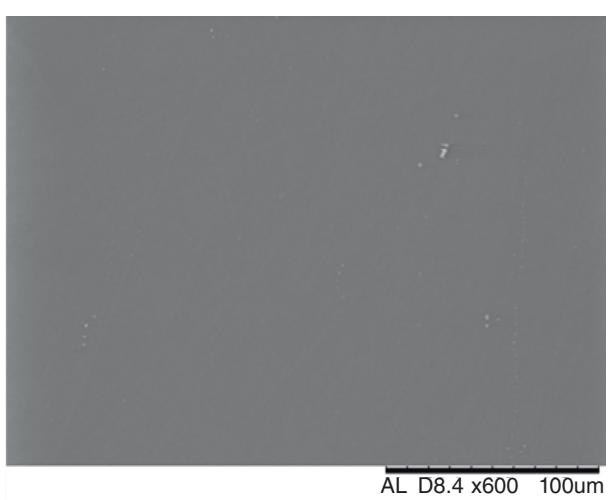

B

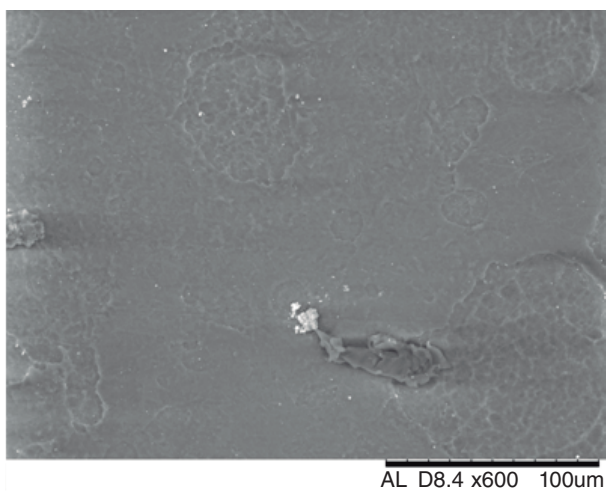

Figura 1: Morfologia superficial das amostras de PC (escala $100 \mu \mathrm{m})$ 
degradação térmica a $50^{\circ} \mathrm{C}$. A variação na morfologia evidencia que ocorreu degradação do PC. Não houve variação na morfologia para o PC a temperatura ambiente. Pode-se inferir que a degradação só ocorreu devido a condição específica para degradação ser de $50^{\circ} \mathrm{C}$ para as condições experimentais adotadas.

O PC em contato com solução aquosa de $\mathrm{NaOH}$ e aquecimento térmico gera os subprodutos bisfenol A (BFA) e carbonato de sódio $\left(\mathrm{Na}_{2} \mathrm{CO}_{3}\right)^{(3)}$. Contudo, as análises por EDS e FRX revelaram que não há presença de átomos de sódio $(\mathrm{Na})$ na superfície degradada. Uma vez que o $\mathrm{Na}_{2} \mathrm{CO}_{3}$ é solúvel em água, este composto possivelmente foi removido durante a lavagem. Assim, as regiões degradadas (Fig. 1b) provavelmente são formadas pelo BFA. Essas regiões apareceram somente sob as condições de $50^{\circ} \mathrm{C}$ e pulverização com solução de $\mathrm{NaOH}$, como mencionado anteriormente.

$\mathrm{O}$ entendimento do comportamento químico do PC fornece uma base fundamental para a pesquisa. O monômero do PC é formado por dois grupos metil $\left(-\mathrm{CH}_{3}\right)$, dois anéis aromáticos ligados ao átomo de carbono e por um grupo carboxílico (-COO) e oxigênio ligados aos extremos de cada anel aromático ${ }^{(3)}$. A Fig. 2 ilustra uma possível equação química útil para o entendimento da degradação do monômero PC, onde n representa o número de repetições do monômero. Os subprodutos BFA e $\mathrm{Na}_{2} \mathrm{CO}_{3}$ são gerados quando o PC sofre degradação térmica na presença de $\mathrm{NaOH}^{(3)}$. O BFA (Fig. 2) apresenta hidroxilas (-OH) ligadas aos anéis aromáticos.

As amostras de PC, antes e após sofrerem degradação térmica, foram expostas à radiação monocromática $\left(\lambda_{\text {exc }}=385 \mathrm{~nm}\right)$ da banda do ultravioleta próximo. $\mathrm{O}$ espectro gerado é apresentado na Fig. 3. Ambas as amostras de PC apresentaram o mesmo

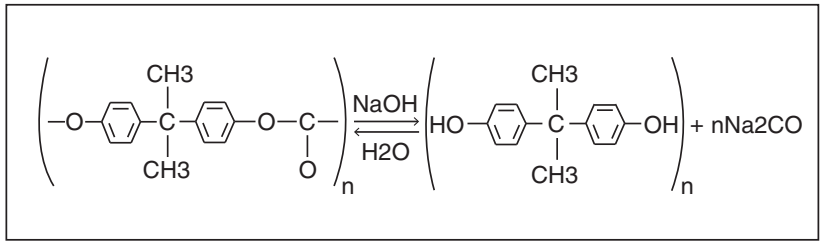

Figura 2: Reação genérica para a degradação do PC.

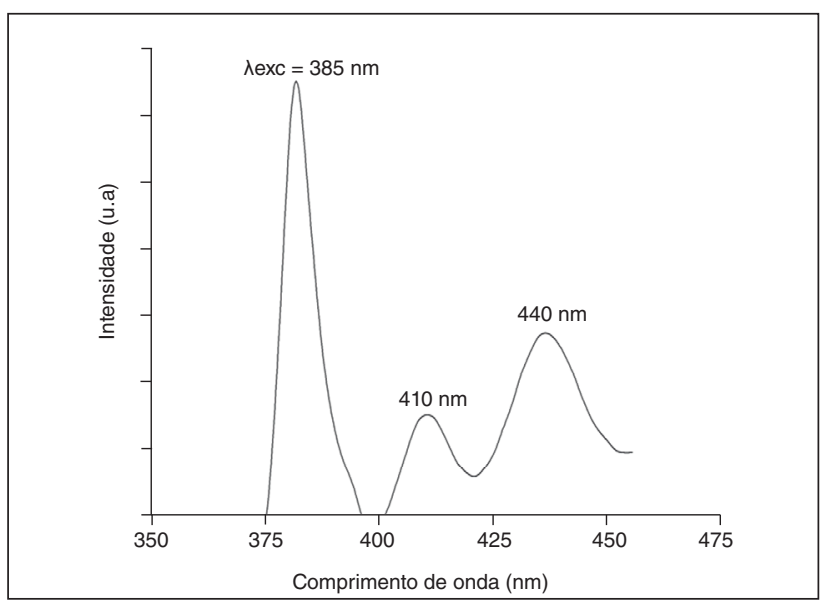

Figura 3: Espectro de excitação e emissão fotoluminescente. espectro de emissão na banda visível (Fig. 3), isso indica que provavelmente a fotoluminescência do PC não seja afetada pela degradação térmica. Analisando o espectro obtido, observou-se que a emissão ocorreu na banda do violeta e na banda do azul, com picos nos comprimentos de onda de $410 \mathrm{~nm}$ e $440 \mathrm{~nm}$, respectivamente.

Mesmo após a degradação, as amostras exibiram o mesmo espectro de emissão fotoluminescente (Fig. 3). Desta maneira, é possível inferir que a atividade fotoluminescente do PC pode ser atribuída a uma parte do monômero do PC. Na Fig. 4 é ilustrado um possível esquema para ajudar a entender a emissão fotoluminescente do PC, antes e após a degradação. A partir do esquema ilustrado na Fig. 4 é assumido que a fração do PC ligada ao grupo carboxílico é responsável pela fotoluminescência.

A fotoluminescência como mencionado anteriormente parece não ser afetada pela degradação do PC. Com base no esquema ilustrado na Fig. 4, possivelmente pode ser atribuída atividade luminescente à fração do PC ligada ao grupo -COO. Essa fração após degradação gera o BFA (Fig. 2). Além disso, a presença de hidrogênio, formando hidroxilas para gerar BFA (Fig. 2), após a degradação do PC, parece não inibir a atividade fotoluminescente da fração do monômero anteriormente ligado ao grupo carboxílico.

Trabalhos sobre a atividade fotoluminescente de materiais a base de carbono, tais como dots de carbono excitados com radiação ultravioleta, relataram emissão dominante da região azul ${ }^{(5-8)}$. A presença de elementos e grupos também influencia a atividade fotoluminescente. Cadeias alcalinas $(-\mathrm{R}-\mathrm{OH})$ ligadas a grafeno induzem a emissão na região azul ${ }^{(6)}$. Por outro lado, grupos amina, devido aos elétrons desemparelhados, induzem emissão na região vermelha ${ }^{(9)}$. Também, há uma melhor fotoluminescência com nitrogênio ligado à superfície de dots de carbono, devido a uma eficiente separação de par elétron-lacuna ${ }^{(10)}$.

Como ilustrado na Fig. 4, há dois caminhos possíveis para a emissão de banda do azul (440 nm) e somente há um único caminho para a emissão na banda violeta $(410 \mathrm{~nm})$. Provavelmente,

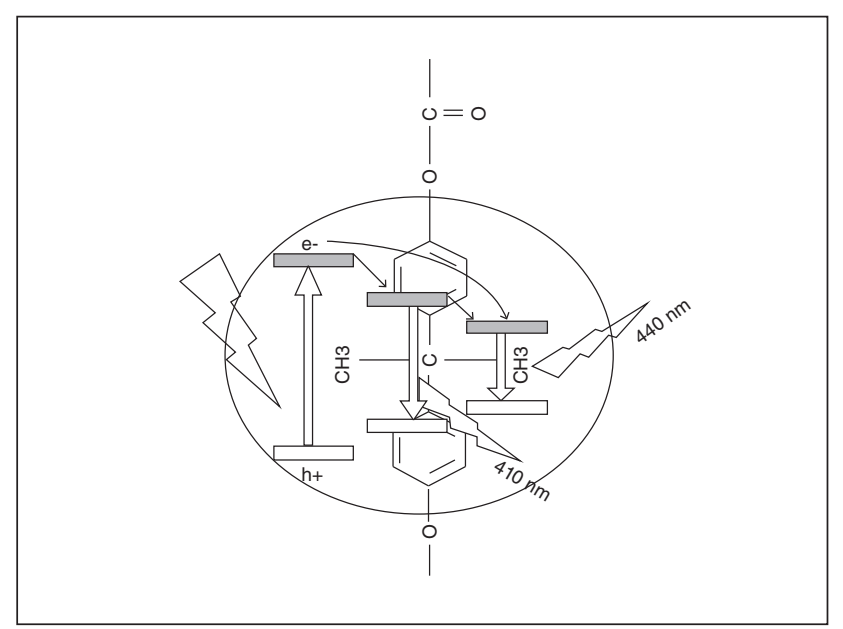

Figura 4: Representação esquemática do mecanismo de fotoluminescência do PC. 
essa vantagem influenciou o espectro de emissão gerado (Fig. 3), onde há maior pico de emissão na banda azul. Possivelmente, os pares de elétrons conjugados nos anéis aromáticos e a presença de hidroxilas (Fig. 2) otimizaram a separação elétron-lacuna resultando numa melhor fotoluminescência na banda azul.

As informações sobre a estabilidade térmica do PC e os efeitos da degradação térmica sobre a propriedade fotoluminescente do PC auxiliam a encontrar novas aplicações para o PC. Adicionalmente, dots de carbono que convertem radiação ultravioleta para luz azul têm sido usado para melhorar a eficiência de células fotovoltaicas ${ }^{(5)}$. A partir deste contexto, o polímero PC, degradado ou não, apresenta potencial para aplicação em células fotovoltaicas, uma vez que exibe a mesma propriedade dos dots de carbono.

\section{CONCLUSÃO}

O processo de despolimerização do PC através da reação de degradação com aquecimento e pulverização da solução de $\mathrm{NaOH}$ foi encontrado ser uma degradação ativa somente sob a condição especifica de $50^{\circ} \mathrm{C}$. Na condição específica de degradação, o PC foi decomposto em BFA e $\mathrm{Na}_{2} \mathrm{CO}_{3}$. Contudo, o polímero PC apresenta similar propriedade fotoluminescente, antes e após a degradação. A fotoluminescência exibida pelo PC após degradação e com base no mecanismo para a degradação e emissão foi assumida ser devido à presença do radical orgânico do BFA ligado às hidroxilas.

A metodologia adotada neste trabalho pode ser de adaptada e melhorada, e assim servir de base para trabalhos futuros destinados ao estudo da degradação baseada na ação de intempéries sobre os polímeros, não sendo restrita somente ao PC. Além de servir de base para pesquisas destinadas ao uso de PC e outros polímeros visando aumentar a eficiência de células fotovoltaicas.

\section{AGRADECIMENTOS}

Os autores agradecem a Coordenação de Aperfeiçoamento de Pessoal de Nível Superior (CAPES), Fundação Cearense de Apoio ao Desenvolvimento Científico e Tecnológico (FUNCAP) e Conselho Nacional de Desenvolvimento Científico e Tecnológico
(CNPq) pelo apoio através da concessão de bolsas de mestrado e doutorado.

\section{REFERÊNCIAS}

1. DELPECH, M.C., COUTINHO, F.M.B., HABIBE, M.E.S., Bisphenol A-based polycarbonates: characterization of commercial samples. Polymer Testing, v. 21, n. 2, 2002, pp. 155 - 161.

2. YAMASAKI, R. S., BLAGA, A., Degradation of polycarbonate sheeting on outdoor exposure relationship between changes in molecular weight and tensile properties. Materiaux et Constructions, v. 10, n. 4, 1997, pp. 197- 204.

3. DEIRRAM, N. et al., Hydrolysis degradation of polycarbonate under microwave irradiation by using design of experiment software. Scientific Research and Essays, v.7, n. 40, 2012; pp. $3399-4405$

4. SARON, C., FELISBERTI, M.I., Influência de colorantes na degradação termo-oxidativa do policarbonato. Revista Matéria, v. 14, n. 3,2009 , pp. $1028-1038$.

5. HUANG, J. J. et al., An easy approach of preparing strongly luminescent carbon dots and their polymer based composites for enhancing solar cell efficiency. Carbon, v. 70, 2014, pp. 190 - 198.

6. ZHU, S. et al., Surface chemistry routes to modulate the photoluminescence of graphene quantum dots: from fluorescence mechanism to up-conversion bioimaging. Advanced Functional Materials, v. 22, n. 22, 2012, pp. 4732 - 4740.

7. LIU, R. et al., Bottom-up fabrication of photoluminescent graphene quantum dots with uniform morphology. J. Am. Chem. Soc., v. 133, n. 39, 2011, 15221 - 15223.

8. DONG, Y. et al., Blue luminescent graphene quantum dots and graphene oxide prepared by tuning the carbonization degree of citric acid. Carbon, v. 50, 2012, pp. 4738 - 4743.

9. JIN, S. H. et al., Tuning the photoluminescence of graphene quantum dots through the charge transfer effect of functional groups. ACS Nano, v. 7, n. 2, 2013, pp. 1239 - 1245.

10. HU, S. et al., Modulation and effects of surface groups on photoluminescence and photocatalytic activity of carbon dots. Nanoscale, v. 5, n. 23, 2013, pp. $11665-11671$.

11. LIMA, F.M. et al., Fluorine-doped tin oxide films by spray pyrolysis using vacuum within nozzle. Rev. Bras. Apl. Vac., v. 34, n.3, 2015, pp. $94-97$. 